\title{
artigo
}

Oliveira, J.G.A.D.; Almeida, L.F.; Andrade, K.B.S.; Paula, V.G.; Pereira, S.R.M.; Marins, A.L.C.; Mesquita, A.M.F.;

Transferências de cuidados entre turnos de enfermagem em uma unidade intensiva

\section{Transferências de cuidados entre turnos de enfermagem em uma unidade intensiva}

\begin{abstract}
RESUMO
Objetivo: Analisar as informações nas transferências de cuidados entre turnos de enfermagem em uma unidade intensiva. Métodos: Estudo descritivo, transversal, realizado em uma UTI geral de um hospital universitário, entre fevereiro e junho de 2017. Incluídas transferências de cuidados entre turnos, realizadas pela equipe de enfermagem. Excluídas aquelas realizadas por docentes e acadêmicos de enfermagem. Os dados foram coletados através de checklist relacionado às informações, categoria profissional, duração da transferência de cuidados, e analisados por estatística descritiva. 0 estudo foi aprovado pelo Comitê de Ética e Pesquisa, número CAAE 64134016.0.0000.5259. Resultados: As informações mais relatadas foram medicações (66\% - 345), valores do balanço hídrico (61,1\% - 310), histórico do paciente $(58,6 \%$ - 306) e dispositivos invasivos $(51,7 \%$ - 270$)$. Conclusão: Informações como dor, enfrentamento da doença e assistência familiar não foram relatadas.
\end{abstract}

DESCRITORES: Comunicação; Enfermagem; Unidade de Terapia Intensiva.

\section{ABSTRACT}

Objective: Analyze information in transfers of nursing shift care in a unit intensive. Methods: Descriptive, cross-sectional study conducted in a general ICU of a university hospital between February and June 2017. Included transfers of care between shifts, carried out by the nursing team. Excluding those performed by professors and nursing students. Data were collected through checklist related to information, professional category, duration of care transfer, and analyzed by descriptive statistics. The study was approved by the Ethics and Research Committee, number CAAE 64134016.0.0000.5259. Results: The most reported information were medications $(66 \%$ - 345), water balance values $(61.1 \%$ - 310), patient history $(58.6 \%$ - 306) and invasive devices (51.7\% - 270). Conclusion: Information such as pain, coping with the disease and family care have not been reported.

KEYWORDS: Communication; Nursing; Intensive Care Unit.

\section{RESUMEN}

Objetivo: Analizar la información en transferencias de cuidados por turnos de enfermería en una unidad intensivo. Métodos: Estudio descriptivo y transversal realizado en una UCl general de un hospital universitario entre febrero y junio de 2017. Incluye transferencias de atención entre turnos, realizadas por el equipo de enfermería. Excluyendo los realizados por profesores y estudiantes de enfermería. Los datos se recopilaron a través de la lista de verificación relacionada con la información, la categoría profesional, la duración de la transferencia de atención y se analizaron mediante estadísticas descriptivas. El estudio fue aprobado por el Comité de ética e investigación, número CAAE 64134016.0.0000.5259. Resultados: La información más reportada fueron medicamentos (66\% - 345), valores de balance hídrico $(61,1 \%$ - 310), historial de pacientes $(58,6 \%$ - 306) y dispositivos invasivos $(51,7 \%$ - 270$)$. Conclusión: Nose hannotificado informaciones como el dolor, la afrontación de la enfermedad y el cuidado familiar.

PALABRAS CLAVE: Comunicación; Enfermería; Unidad de Cuidados Intensivos.

RECEBIDO EM: 28/10/2019 APROVADO EM: 29/10/2019

\section{Jéssica Grativol Aguiar Dias de Oliveira \\ Especialista em Enfermagem em Terapia Intensiva na Modalidade de Residência.}

\section{Luana Ferreira de Almeida}

Professora Adjunta da Faculdade de Enfermagem. Universidade do Estado do Rio de Janeiro. Doutora em Educação em Ciências e Saúde

\section{Karla Biancha Silva de Andrade}

Professora Adjunta da Faculdade de Enfermagem. Universidade do Estado do Rio de Janeiro. Doutora em Enfermagem 


\section{Vanessa Galdino de Paula}

Professora Assistente da Faculdade de Enfermagem. Universidade do Estado do Rio de Janeiro. Doutora em Enfermagem e Biociências

\section{Sandra Regina Maciqueira Pereira}

Professora Adjunta da Faculdade de Enfermagem. Universidade do Estado do Rio de Janeiro. Doutora em Enfermagem

\section{Ana Lúcia Cascardo Marins}

Professora Assistente da Faculdade de Enfermagem. Universidade do Estado do Rio de Janeiro. Mestre em Enfermagem

\section{Ayla Maria Farias de Mesquita}

Professora Assistente da Faculdade de Enfermagem. Universidade do Estado do Rio de Janeiro. Mestre em Enfermagem

\section{INTRODUÇÃO}

A transferência de cuidado corresponde a uma atividade que exige dos profissionais comprometimento com a continuidade segura da assistência, visto que a qualidade dessas informações colabora com a Sistematização da Assistência de Enfermagem - SAE ${ }^{(1)}$. No entanto, muitas informações são negligenciadas pela equipe durante esse momento, fato que potencializa a ocorrência de eventos adversos, comprometendo a segurança do paciente ${ }^{(1)}$.

Diante do contexto de trabalho em terapia intensiva e da gravidade dos pacientes, a comunicação entre os profissionais de enfermagem, neste ambiente, deve ocorrer de modo a não deixar lacunas nos dados transmitidos ${ }^{(2)}$. Neste sentido, e buscando a prevenção de falhas, as transferências de cuidado devem permitir que os profissionais tenham conhecimento da evolução clínica do paciente, das intercorrências, pendências para o próximo turno de trabalho, dentre outros. Assim, as transferências de cuidado devem conter todas as informações indispensáveis para continuidade da assistência, e devem ser transmitidas de forma clara, precisa, objetiva, evitando-se más interpretações ${ }^{(1,3)}$.

Recomendações relacionadas à transferência interna e passagem de plantão dos pacientes têm sido contempladas em diferentes estudos, na busca de melhorias à segurança do paciente no contexto hospitalar ${ }^{(4,5)}$. Informações relativas à evolução do estado de saúde dos pacientes, encaminhamentos, pendências, entre outros, correspondem a importantes elementos e possibilitam a reflexão sobre os aspectos
[...]

Informações

relativas à evolução

do estado de saúde

dos pacientes,

encaminhamentos,

pendências, entre

outros, correspondem

a importantes

elementos e

possibilitam a

reflexão sobre

os aspectos

que envolvem a

transferência de

cuidados entre

equipes.

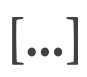

que envolvem a transferência de cuidados entre equipes. É importante assegurar que os profissionais envolvidos não tenham dúvidas e reconheçam a importância da clareza na troca de informações dos dados para a sequência adequada e cuidados necessários para cada paciente ${ }^{(6)}$.

A partir dessas reflexões, surgiu a seguinte questão norteadora: Quais as informações relacionadas às transferências de cuidados de enfermagem em uma unidade intensiva? Dessa forma, este estudo teve como objetivo analisar as informações nas transferências de cuidados entre turnos de enfermagem em uma unidade intensiva.

\section{METODOLOGIA}

Estudo descritivo, transversal, realizado em uma UTI geral de um hospital universitário do Rio de Janeiro de grande porte. A unidade possui capacidade de dez leitos, admite indivíduos adultos, com perfil clínico e cirúrgico.

Foram incluídas no estudo as transferências de cuidados entre turnos realizadas do serviço noturno para o diurno e vice-versa, realizadas por enfermeiros, técnicos e residentes de enfermagem. Excluídas aquelas realizadas por docentes e acadêmicos de enfermagem. Os dados foram coletados no período de fevereiro a junho de 2017, através de checklist com itens relacionados às informações descritas na literatura e preconizadas para a transferência de cuidados, categoria profissional e duração da transferência.

Foram organizados e tabulados em planilhas do Programa Excel para tratamento estatístico descritivo, a fim de avaliar frequências absolutas e relativas. O estudo 


\section{artigo}

Oliveira, J.G.A.D.; Almeida, L.F.; Andrade, K.B.S.; Paula, V.G.; Pereira, S.R.M.; Marins, A.L.C.; Mesquita, A.M.F.; Transferências de cuidados entre turnos de enfermagem em uma unidade intensiva.

foi aprovado pelo Comitê de Ética em Pesquisa sob o número do parecer CAAE 64134016.0.0000.5259.

\section{RESULTADOS}

Foram analisadas 522 transferências de cuidado entre turnos de enfermagem, sendo a maioria delas analisada no período diurno $(80,0 \%$ - 48). Grande parte das transferências de cuidados foi feita pelo enfermeiro plantonista $(88,0 \%-53)$ e o tempo médio de duração de cada uma foi de 21 minutos. As informações transferências de cuidados entre turnos de enfermagem estão descritas na Tabela 1.

\section{DISCUSSÃO}

Neste estudo, a descrição das medicações e infusões contínuas mostrou ser de grande preocupação da equipe, estando presente em 66,0\% (345) das transferências, contrapondo-se com outra pesquisa ${ }^{(7)}$ que evidenciou que os enfermeiros intensivistas repassaram com menos frequência as informações relacionadas a medicamentos.

A informação sobre os medicamentos utilizados pelo paciente é relevante tendo em vista a prevenção de eventos relacionados à terapia medicamentosa. Neste momento, podem ser conferidos, além dos tipos de medicamentos, as doses e vias utilizadas, sendo um momento oportuno para a correção de falhas relacionadas ao processo terapêutico.

Tabela 1. Informações presentes durante as transferências de cuidados entre turnos de enfermagem. Rio de Janeiro, RJ, Brasil, 2017 ( $n=522)$

\begin{tabular}{lcc}
\multicolumn{1}{c}{ INFORMAÇÕES } & N & $\%$ \\
Medicações & 345 & 66,0 \\
Balanço hídrico & 319 & 61,1 \\
Histórico & 306 & 58,6 \\
Dispositivos invasivos & 270 & 51,7 \\
Integridade e/ou lesões de pele & 200 & 38,3 \\
Cuidados de enfermagem & 135 & 25,8 \\
Proposta terapêutica & 118 & 22,6 \\
Pendências & 83 & 16,0 \\
Alergias & 29 & 5,5
\end{tabular}

O valor do balanço hídrico foi relatado em $61,1 \%$ (319) dos casos. Tal resultado se opõe ao estudo ${ }^{(8)}$ que buscou descrever a atuação dos profissionais de enfermagem na realização da passagem de plantão, a partir de uma revisão de literatura. A pesquisa mostrou que $28,6 \%$ mencionou informações sobre quadro clínico e cuidados prestados, além de $23,8 \%$ sobre intercorrências com o paciente.

O estado do paciente durante o período em que foi assistido e as informações geradas durante esse momento buscam subsidiar o planejamento da assistência durante o próximo plantão, possibilitando que a meta para o cuidado a ser prestado seja estabelecida. Por isso, é de grande valia o conhecimento de informações que norteiem a assistência de enfermagem, sobretudo na terapia intensiva, onde muito pacientes encontram-se hemodinamicamente instáveis.

O histórico do paciente foi apresentado em 58,6\% (306) das transferências de cuidados. Este deve conter dados para identificação, data de admissão no hospital e na UTI, bem como sua procedência, além da história social e familiar ${ }^{(9)}$. A identificação do paciente, com nome, data de nascimento e número de registro na instituição é preconizada na unidade estudada. No entanto, percebe-se uma fraca cultura de segurança do paciente quando se observam os dados apresentados. Além disso, a história social e familiar do paciente permite a identificação de outras necessidades e seu acompanhamento para além do físico, mas também para as demandas psicossociais apresentadas.

Os relatos quanto à presença de dispositivos invasivos foram também destacados neste estudo (51,7\% - 270), divergindo de outra pesquisa ${ }^{(10)}$ que evidenciou a necessidade de informações completas em relação à presença de cateteres e/ou sondas. Verificou-se uma atenção especial aos dispositivos, que são muito utilizados pelos pacientes na terapia intensiva para suporte hemodinâmico e nutricional. Os cateteres e sondas permitem, no paciente em estado grave, não só a infusão de medicamentos e dietas, mas também a eliminação de líquidos como diurese e conteúdo gástrico.

As informações sobre a integridade da pele ou presença de lesões foram citadas em $38,3 \%$ (200) das transferências. Divergindo desse dado, as lesões de pele estiveram dentre os eventos adversos mais notificados de um hospital geral ${ }^{(11)}$. Nesse aspecto, devem ser consideradas algumas recomendações sempre que necessário e possível para prevenir lesões desse tipo ${ }^{(12)}$. Isso justifica-se, pois as lesões de pele são consideradas eventos adversos, ou seja, uma complicação indesejável e pode levar o paciente a adquirir infecção. Costumam aumentar o tempo de permanência do paciente na instituição e os custos hospitalares, pela necessidade imediata de seu tratamento.

Os cuidados de enfermagem foram relatados em 25,8\% (135) dos casos. Corroborando com o presente estudo, uma pesquisa ${ }^{(13)}$ mostrou que entre as principais falhas estão omissões de informações das condições críticas dos pacientes 19,2\% e omissões sobre o plano de cuidados $14,2 \%$. Tais dados mostram-se preocupantes, visto que é a partir do processo de enfermagem que é possível a identificação de necessidades dos pacientes, a implementação dos cuidados prestados e avaliação da resposta do paciente aos mesmos.

Quanto às propostas terapêuticas, em $22,6 \%$ (118) dos casos destacaram-se medidas de conforto e assistência em cuidados paliativos, transferência para outra unidade, abordagens cirúrgicas, necessidade de 
diálise e demais terapêuticas implantadas de acordo com a doença do paciente. Durante as transferências de cuidado devem ser abordadas informações referentes ao estado dos pacientes, tratamentos, assistência prestada, intercorrências, pendências e situações referentes a fatos específicos da unidade de internação que merecem atenção ${ }^{(14)}$. Juntamente às propostas terapêuticas, em 16,0\% (83) das transferências foram enunciadas as pendências do plantão, destacando-se a necessidade de realizar a reposição dos mesmos, a fim de garantir insumos para o próximo plantão.

A informação menos referida pelos profissionais de enfermagem tratou de alergias apresentadas pelos pacientes, principalmente a alergia medicamentosa (5,5\% - 29). Os principais tipos de alergia encontrados em pacientes adultos/idosos correspondem a dermatite de contato, urticária e, principalmente, a hipersensibilidade medicamentosa ${ }^{(15)}$. Deste modo, é imprescindível atentar para esse aspecto, visto que as reações medicamentosas podem apresentar graus de gravidade diferenciados, causando desde leve desconforto, como prurido, e até sinais e sintomas mais graves provocados por reações anafiláticas, podendo progredir para choque anafilático e óbito ${ }^{(16)}$.

\section{CONCLUSÃO}

Este estudo permitiu analisar as informações durante transferências de cuidados entre turnos de enfermagem em uma unidade intensiva. Verificou-se que os profissionais valorizaram dados mais objetivos, focando em medicações, uso dos cateteres, demais dispositivos e pele. Observou-se que não se aprofundaram em dados subjetivos referentes aos pacientes, que também são importantes para o entendimento de alguns sinais e sintomas e para o planejamento do cuidado de enfermagem, como: queixas dos pacientes, dor, interação família-paciente, enfrentamento da doença, medos relacionados ao diagnóstico e tratamento.

Neste contexto, a proposta sugerida a partir deste estudo consiste na utilização de instrumento que busque contemplar as informações descritas na literatura, como o registro de alergias. $\mathrm{O}$ estudo mostrou-se relevante para detectar lacunas na comunicação durante as a transferências entre turnos de enfermagem, sobretudo, quando se trata de pacientes graves. Nesse sentido, proporcionou reflexão sobre as principais informações a serem relatadas sobre pacientes e as prioridades durante esse momento de comunicação entre profissionais.

\section{REFERÊNCIAS}

1. Bueno BRM, Moraes SS, Suzuki $K$, Gonçalves FAF, Barreto RASS, Gebrim CFL. Caracterização da passagem de plantão entre o centro cirúrgico e a unidade de terapia intensiva. Cogitare Enferm. 2015; 20(3):512-518.

2. Empaire PP, Amara ACKB. 0 que todo intensivista deveria saber sobre a passagem de plantão na unidade de terapia intensiva. Rev Bras Ter Intensiva. 2017; 29(2):121-123.

3.Nogueira AWS, Rodrigues MCS. Comunicação efetiva no trabaIho em equipe em saúde: um desafio para a segurança do paciente. Cogitare Enferm. 2015; 20(3):636-640.

4. Minuzzi AP, Salum NC, Locks MOH, Amante LN, Matos E. Contribuições da equipe de saúde visando à promoção da segurança do paciente no cuidado intensivo. Escola Anna Nery. 2016; 20(1):121-129.

5. Silva SG, Nascimento ERP, Hermida PMV, Sena AC, Klein TCR, Pinho FM. Checklist para passagem de plantão de pacientes em pós-operatório imediato na admissão em terapia intensiva. Enferm. Foco. 2016; 7(1).

6. Oliveira MC, Rocha RGM. Reflexäo acerca da passagem de plantäo: implicaçöes na continuidade da assistência de enfermagem. Enfermagem Revista. 2015; 2(19):191-198.

7. Gonçalves MI, Rocha PK, Anders JC, Kusahara DM, Tomazoni A. Communication and patient safety in the change-of-shift nurssing report in neinatal intensive care units. Texto Contexto-Enferm. 2016; 25(1):e2310014.

8. Nogueira SR, Oliveira AML, Devezas LSCS. Relevant aspects in the nursing professional's performance during the shift. Brazilian
Journal of health Review. Braz. J. Hea. 2019; 2(4):2418-2437.

9. Spooner AJ, et al. Desenvolvendo um conjunto mínimo de dados para a entrega do líder da equipe de enfermagem na unidade de terapia intensiva: um estudo em grupo de foco. Australian College of critical care nurses. 2018; 31(1):47-52.

10. Almeida FAV, Costa MLAS. Passagem de plantão na equipe de enfermagem: um estudo bibliográfico. Arq Med Hosp Fac Cienc Med Santa Casa São Paulo. 2017; 62(2):85-91.

11. Xelegati R, Gabriel CS, Dessotte CAM, Zen YP, Évora YDM. Adverse events associated to the use of equipment and materials in nursing care of hospitalized patients. Rev Esc Enferm USP. 2019; 53:e03503.

12. National Pressure Ulcer Advisory Panel. Prevention and treatment of pressure ulcers: quick reference guide. Perth: Cambridge Media; 2014.

13. Thomas MJW, Schultz TJ, Hannaford N, Runciman WB. Failures in transition: learning from incidents relating to clinical handover in acute care. J Healthc Qual. 2013; 35(3).

14.Lima AM, Silva ER. Sistematização da passagem de plantão em unidade de terapia intensiva: proposta de instrumento de registro. Revista Recien. 2017; 7(20):48-57.

15. Wong A, Malvestiti AA, Hafner MGS. Síndrome de Stevens-Johnson e necrólise epidérmica tóxica: uma revisão. Revista da Associação Médica Brasileira. 2016; 62(5): 468-473.

16. Pedreirinho A, Godinho H, Pinto M, Correia P, Mendes F, Marques MC. Alarma de fatiga en la seguridad del paciente: revisión sistemática. Revista ibero-americana de salud y envejecimiento. 2016; 2(2):544-562. 